\section{Headaches as somatoform disorders in children and adolescents}

\section{Ewa Emich-Widera, Beata Kazek, Barbara Szwed-Białożyt, llona Kopyta, Anna Kostorz}

Department of Pediatrics and Child Neurology, Medical University of Silesia, Katowice, Poland

\section{Abstract}

Somatoform disorders are often the main cause for seeking professional advice and performing a number of specialist checks. The aim of the study was to determine the frequency of somatoform disorders in the form of headaches in children and adolescents neurologically diagnosed and the risk factors thereof. Analysis of the biological and situational risk factors were established. Somatoform disorders were diagnosed in 27 out of 276 children with headaches. We concluded that in the differential diagnosis of headaches, somatoform headaches should not be omitted as every $10^{\text {th }}$ patient in the developmental age diagnosed on the neurological ward because of headache shows signs of somatoform headaches. In diagnostically difficult cases it is recommended that analysis of biological and situational risk factors be performed with special attention paid to chronic disease of the patient and/or in his immediate family, the patient's psychological disorders and dysfunctional or low social status families. The creation of separate criteria for somatoform disorders of the developmental age should be considered.

\section{Introduction}

Headaches affect more than half of the people in the developmental age - single headache incident has been reported by $54-60 \%$ of children and adolescents (including more than $60 \%$ at the age of 14 ), while recurring ailments have been noticed by almost $7 \%$ of children and adolescents.1,2 Headache is one of the symptoms which are the most often reported by patients to their doctor, also to child neurologists. Migraine and tension type headaches (TTH) remain the most common type of headache in the developmental age group. .,3,4 $^{2}$

Somatoform headaches are significantly less common. The International Classification of Diseases (ICD-10) includes them in chapter F Classification for Mental and Behavioural Disorders - neurotic stress-related and somatoform disorders (F40-F48). The disorders encompass pain and other complex somatic symptoms related to the functioning of the autonomic nervous system that last at least for 24 months. In some of the patients the abovementioned problems coexist with conversion disorders, characterized by partial or complete loss of normal interaction between memories from the past, sense of identity, direct impressions and voluntary movements. It is typical that the symptoms of conversion disorders are involuntary. They are related to traumatic situations beyond the patient's understanding and which they are incapable of dealing with. Both somatoform and conversion disorders are the reasons why patients are seeking professional help and undergoing a number of specialist examinations.

According to the literature and our own observations a number of biological and situational risk factors dealing with somatoform disorders were collected (Table 1).

\section{Materials and Methods}

To determine the frequency of somatoform disorders in the form of headaches in children and adolescents hospitalized in the Department of Pediatrics and Child Neurology and the risk factors thereof.

This thesis is a retrospective assessment based on analysis of the documentation of 276 patients suffering from headaches and diagnosed during the last 6 years at the Department of Pediatrics and Child Neurology of the Medical University of Silesia in Katowice. The study group consisted of 27 patients with somatoform headaches (Sc group), including 18 patients with only somatoform symptoms (sub-group $S$ ) and 9 patients presenting both somatoform and conversion symptoms (sub-groups $\mathrm{S}+\mathrm{K}$ ). The reference group was 144 patients with TTH. The patients were included in the analyzed groups based on the ICD-10 diagnostic criteria: on the basis of the results of physical examination, anamnesis, results of additional examinations and consultations, which of course included psychological and psychiatric.

Patients with diagnosed coexistence of other types of headaches, those whose symptoms occurred as a result of physical trauma or extreme stress (for example life threatening situations causing posttraumatic stress disorders) or patients intoxicated with psychoactive drugs and those with the Munchausen syndrome and Munchausen syndrome by proxy were excluded.

The diagnosis of histrionic features was made by clinical interview according to the ICD 10 criteria.

Biological and situational risk factors caus-
Correspondence: Beata Kazek, Department of Pediatrics and Child Neurology of the Medical University of Silesia 40 - 752 Katowice ul. Medyków 16; Poland. E-mail: beakazek@op.pl

Key words: somatoform disorders, tension type headaches, children, adolescents.

Conflict of interests: the authors report no conflict of interests.

Received for publication: 26 September 2011.

Revision received: 27 February 2012.

Accepted for publication: 27 February 2012

This work is licensed under a Creative Commons Attribution NonCommercial 3.0 License (CC BYNC 3.0).

(C) Copyright E. Emich-Widera et al., 2012

Licensee PAGEPress, Italy

Mental Illness 2012; 4:e9

doi:10.4081/mi.2012.e9

ing the analysed disorders were established.

The results were statistically analysed using Fisher's exact test and the Chi-square test with Yates's correction. Significance level $P(\alpha)$ $<0.05$.

\section{Results}

The results are presented in tabular registers (Supplementary Tables 1-6). Obtained data indicate that female patients are dominant in all groups (Supplementary Table 1). The majority of patients aged between 15 and 17 years old were in the study group, while in the reference group the distribution of age was equal (Supplementary Table 2). Supplementary Table 3 contains different symptoms accompanying headaches. In the group of patients with somatoform disorders, joint and limb pains and stomach ache very often occurred. Even $30 \%$ of patients with somatoform and conversion disorders suffer from dizziness, joint and limb pains, stomach aches, heart palpitations, back aches and chest pains. In the group of patients with TTH there was no one symptom which occurred in large groups of patients. Supplementary Table 4 shows the percentage of patient in the respective groups of disorders, depending on the duration symptoms. The period of time between the onset of symptoms and the final diagnosis varies significantly in the respective groups (Chi-square test with Yates's correction). As agreed upon for the thesis, the biological risk factors of somatoform, conversion and tension headaches were analysed. In the analysed patients we observed the coexistence of organic chronic disease - irrespective of whether or not the patient belonged to the study group (S, 
$\mathrm{S}+\mathrm{C}, \mathrm{T})$. The following were reported the most often: posture defects, allergy (including asth$\mathrm{ma}$ ) and disorders of the urinary system and of the heart rhythm.

Analysis of the biological risk factors revealed that in the group of patients with somatoform headaches the following were observed more often than in the patients with tension headaches - histrionic features $(\mathrm{P}<0.005)$, coexisting chronic disease $(\mathrm{P}<0.005)$, disorder of the bioelectric brain activity $(\mathrm{P}<0.005)$, coexisting psychological disorders $(\mathrm{P}<0.0001)$ and abnormal body mass index (BMI, $\mathrm{P}<0.005)$. Analysis of the symptoms reported in the groups indicates that 9 patients with somatoform disorders showed symptoms imitating ailments occurring in their families. In 6 cases the ailments included headaches and dizziness (occurred in their mothers), one child was presented with pseudo-epileptic seizures (uncle suffering from epilepsy), one child with spinal pain syndrome (father with prolapsed disc) and one patient with symptoms of pseudo-deficits in the central nervous system (the patient's sister had been diagnosed with a brain tumour).

Analysis of the situational risk factors (Supplementary Table 6) revealed that in the group of patients with somatoform headaches the following were observed more often: occurrence of chronic diseases in the patient's immediate environment $(\mathrm{P}<0.005)$, dysfunctional family $(\mathrm{P}<0.001)$ and low socio-economic status $(\mathrm{P}<0.005)$ (Fisher's exact test).

Difficulties concerning learning and adapting to a new environment, and place of living were not significantly relevant for the analysis of differences between the respective groups.

Table 1. Risk factors of somatic disorders.

\begin{tabular}{l} 
Biological factors \\
Abnormal body mass index \\
Histrionic features of the developing personality* \\
\hline Bioelectric brain activity disorders \\
Coexistence of organic chronic disease \\
(excluding psychiatric disorders) \\
\hline Below average IQ \\
Past head trauma \\
\hline Coexistence of psychiatric disorders \\
Situational factors \\
Low socio-economic status \\
Dysfunctional family \\
Chronic diseases including psychiatric \\
ones occurring in the child's immediate family. \\
Learning difficulties \\
\hline Peer relation difficulties \\
Place of living: city; countryside \\
* refers to adolescents and preadolescents.
\end{tabular}

\section{Discussion}

Patients with TTH were the reference group for patients suffering from either only somatoform or both somatoform and conversion headaches. The reason for that was because patients with TTH often presents emotional distress (very often accompanied by increased emotional tension, neurotic syndrome or mood swings). Additionally TTH remain the most common form of primary headaches in the population of children and adolescents. ${ }^{1}$

In children and adolescents the prevalence of headaches rose with increasing age. Before the age of twelve the rate of headaches is comparable in both sexes, but after puberty girls with headaches outnumber boys. ${ }^{4}$ In the developmental age likewise sex does not seem to be relevant for the analysis of somatoform disorders apart from the symptoms imitating the morphology of neurological disorders, where the prevalence of girls was observed (2:1). This has been observed in the youngest age groups with a rising trend in preadolescents and adolescents. ${ }^{3}$ In each of the analysed groups of patients the domination of females was noticeable and especially within the subgroup of patients with conversion and somatoform disorders (77.8\%) (Supplementary Table 1).

Generally patients with headache more often have other medical conditions. ${ }^{4}$ In the group of patients with TTH age did not seem to be relevant, while in the study group the majority of patients were between the age of 12 and 17 (Supplementary Table 2). This would confirm the intensification of somatization in the form of headaches from the age of 12.3

The frequency of somatisation in the population of children and adolescents is estimated at $10-25 \% .5$ Manifestation of psychosomatic disorders is often polisymptomatic. The results of analyses done by Campo et al. showed that patients report the following symptoms: (starting with the most frequent) recurring pain, including headaches (10-30\%), stomach aches (10-25\%), limb pains - also the so called growing pains (5-20\%), chest pains (7-15\%), fatigue, dizziness, pseudo-epileptic seizures and other. ${ }^{3}$ The main symptom in our analysed group was of course headaches, but the prevalence of stomach ache, joint and limb pain and dizziness were also high (Supplementary Table 3).

The diagnosis of conversion disorders in children is more difficult than in adult patients, so wrong diagnoses are not uncommon. Stone et al. analysed the number of diagnostic mistakes among adult patients with conversion disorders and it turned out that in $4 \%$ of cases further observations revealed somatic origin of the disorders. ${ }^{6}$ Diagnostic mistakes in the group of patients in the developmental age are estimated between $0-17 \%{ }^{7}$
In the case of our patients with somatoform or somatoform and conversion disorders the period of time between the breakout of the disease and final diagnosis was significantly longer than for patients from the control group, which may indicate that their GPs had diagnostic problems - more than $30 \%$ of patients had to wait three or more years for their final diagnosis (Supplementary Table 4). It has to be emphasized that acceptance of diagnosis of somatoform and conversion disorders is sometimes difficult for the patients and their families. It is often accompanied by some form of attachment to the somatic symptoms as the patient expects them to have a neurological and not a psychological origin. ${ }^{8}$ Therefore in our patients we cannot exclude that it was the posture of the parents that affected the diagnostic process of the child. However, the long waiting time for final diagnosis may be also caused by the criteria for recognition of somatoform disorders - according to the ICD10 classification the minimum period for which symptoms must last is 24 months. Maybe this is a reason for creating a separate classification for the developmental age, even more so because in the case of conversion disorders quick diagnosis and explaining to the patient and his family the nature of the disease has a positive impact on the prognosis. ${ }^{9}$ Therapy and problem solving should start before reaching maturity as conversion disorders have better prognosis in children and adolescents. ${ }^{7,10}$

Lateef et al. found that there is an association between headache and being overweight in American children. ${ }^{4}$ In our research patients with conversion and somatoform disorders had abnormal BMI (mainly lower BMI) more often than patients from the control group $(\mathrm{P}<0.005)$. In the available relevant literature the authors came across only one work which included BMI in the characteristics of the experimental group composed of patients with somatoform disorders. But in this work BMI was similar in both groups. ${ }^{11}$ Specific personality disorders in the form of histrionic disorders are seen significantly often in patients with eating disorders such as anorexia or bulimia. ${ }^{2,13}$ Histrionic features of developing personality were observed more often in the study group (Supplementary Table 5). This may suggest common a etiology of abnormal BMI as an indicator of eating disorders and histrionic personality disorders diagnosed in the patients from the experimental groups. It is worth pointing out that histrionic personality features are not only favourable to forming, but also to maintaining conversion symptoms. ${ }^{14}$

Adults with psychosomatic disorders experienced health problems in their childhood, including hospitalization. Similarly, disorders that occur in early childhood may predeter- 
mine conversion or somatoform condition in the developmental age. ${ }^{3}$ Our patients were also diagnosed with existing chronic organic diseases (Supplementary Table 5). In a way somatoform disorders with or without the conversion element may be the continuation of somatic health problems. Awareness of existing health problems and the diagnostic process (examinations, uncertain diagnosis) may increase the sense of illness. ${ }^{3}$ In the medical history of patients with conversion symptoms in the form of pseudo-epileptic seizures and head traumas very often precede the occurrence of the symptoms. ${ }^{8}$ However this factor is not vital for our experimental group (Supplementary Table 5).

In the group with somatoform disorders chronic diseases in the families were more common (mainly in mothers) (Supplementary Table 6). Every third patient presented symptoms imitating the morphology of neurological disorders observed in his family. Slater et al.,15 Ercan et al. ${ }^{10}$ and Campo et al. ${ }^{3}$ came to similar conclusions. According to Gulhati et al. ${ }^{16}$ parents of children diagnosed because of chronic headaches suffer more often from mainly neurological diseases which intensify three years before the occurrence of health problems in their children. Very often their maternal grand-mothers are dead and the mothers report loneliness, they don't have faith in medicine and are overanxious (Gulhati et al. 1998). ${ }^{16}$ An additional difficulty is caused by the family's predisposition to somatisation disorders (mainly on the part of the mother). Ercan et al. discovered that $43 \%$ of mothers whose children were diagnosed with conversion disorders also fulfilled the criteria for conversion and $39.5 \%$ of them suffered from other types of psychiatric disorders - mainly anxiety. 10

Another situational factor that affects the occurrence of this type of disorders is a dysfunctional family (incomplete or foster family, uneasy atmosphere at home, abnormal intrafamilial relationships) and quite often low socioeconomic status related with the abovementioned group of factors (Supplementary Table 6). The results we obtained are comparable with those of Campo et al., ${ }^{3}$ Albrecht et al.,5 Ercan et al., ${ }^{10}$ Zeharia et al. ${ }^{13}$ Turkish authors suggest that the countryside as a place of living is significantly favourable for the occurrence of somatoform disorders - however our analyses do not confirm this theory.10 Risk factors analysed in patients with conversion disorders (for example: coexisting chronic disease, coexisting psychological disorders, abnormal BMI, occurrence of chronic diseases in the patient's immediate environment, dysfunctional family and low socio-economic status) may be perceived as stress-inducing. Chronic stress in patients at the developmental age is demonstrated by disorders related to physiological processes (concerning sleep, appetite, mood), but also recurring somatic symptoms - mainly pain (headache, stomach ache, muscular/skeletal pains), and sometimes skin lesions (urticaria). The abovementioned symptoms seem to indicate dysfunction of the central and peripheral nervous system as well as the endocrine and immunology system. The possibility of the occurrence of passive organic lesions also seems to be vital. Maybe psychoneuroimmunology will give all the answers about this group of disorders. 15

The diagnosis of somatisation disorders requires a holistic assessment of the patient's situation, not only in terms of coexisting diseases but also the functioning of his family, often with the history of chronic disease or disability of one of the parents.

\section{Limitations}

The studied groups are different in size, which to some degree limits the interpretation of the result.

\section{Conclusions}

In the differential diagnosis of headaches, somatoform headaches should not be omitted as every $10^{\text {th }}$ patient in the developmental age diagnosed on the neurological ward because of headache shows signs of somatoform headaches. In diagnostically difficult cases it is recommended that analysis of biological and situational risk factors be performed with special attention paid to the chronic disease of the patient and/or in his immediate family, the patient's psychological disorders, dysfunctional or low social status families. The creation of separate criteria for somatoform disorders of the developmental age should be considered.

\section{References}

1. Kröner-Herwig B, Heinrich M, Morris L. Headache in German children and adolescents: a population - based epidemiological study. Cephalgia 2007;27:519-27.

2. Roth-Isigkeit A, Thyen U, Stöven $\mathrm{H}$, et al. Pain among children and adolescents: restriction in daily living and triggering factors. Pediatrics 2005:115:152-62.

3. Campo JV, Fritsch SL. Somatisation in children and adolescents. J Am Acad Child Adolesc Psychiatry 1994;33:1223-35.

4. Lateef TM, Merikangas KR, He J, et al. Headache in National Sample of American Children: Prevalence and comorbidity. J Child Neurol 2009;24:536-43.

5. Albrecht S, Naugle AE. Psychological assessment and treatment of somatization: adolescents with medically unexplained neurologic symptoms. Adolesc Med 2002;13:625-41.

6. Stone J, Smyth R, Carson A, et al. Systematic review of misdiagnosis of conversion symptoms and hysteria. BMJ 2005;331:989.

7. Jans T, Schneck-Seif S, Weigand T. Long term outcome and prognosis of dissociative disorder with onset in childhood or adolescence. Child Adolesc Psych Mental Health 2008;2:19.

8. Laederach-Hofmann K, Rüddel H, Mussgay L. Pathological baroreceptor sensivity In patients suffering from somatisation disorders: do they correlate with symptoms? Biol Psychol 2008;79:243-9.

9. Jáuregui Lobera I, Santiago Fernández MJ, Estébanez Humanes S. Eating behaviour disorders and personality. A study using MCMI-II. Aten Primaria 2009;41:201-6. [Article in Spanish].

10. Lucka I, Cebella A. Characteristics of the forming personality in children suffering from anorexia nervosa. Psychiatria Pol 2004;38:1011-8. [Article in Polish]

11. Simeon D, Guralnik 0, Knutelska M, Schmeidler J. Personality factors associated with dissociation: temperament, defenses, and cognitive schemata. Am J Psychiatry 2002;159:489.

12. Owczarek K. Conversion disorders in clinical practice. Advances of Psychiatry and Neurology 2002;11:131-8.

13. Zeharia A, Mukamel M, Carel C, et al. Conversion reaction: management by the paediatrician. Eur J Pediatr 1999;158:1604.

14. Ercan ES, Varan A, VeznedaroGlu B. Associated features of conversion disorder in Turkish adolescents. Pediatr Int 2003;45:150-5.

15. Slater JA. Deciphering emotional aches and physical pains in children. Pediatr Ann 2003;32:402-7. 